\title{
p66Shc regulates renal vascular tone in hypertension-induced nephropathy
}

\author{
Bradley Miller, ${ }^{1}$ Oleg Palygin, ${ }^{2}$ Victoriya A. Rufanova, ${ }^{1}$ Andrew Chong, ${ }^{1}$ Jozef Lazar, ${ }^{2}$ Howard J. Jacob, ${ }^{2}$ David Mattson, ${ }^{2}$ \\ Richard J. Roman, ${ }^{2}$ Jan M. Williams, ${ }^{2}$ Allen W. Cowley Jr., ${ }^{2}$ Aron M. Geurts, ${ }^{2}$ Alexander Staruschenko, ${ }^{2}$ \\ John D. Imig, ${ }^{3}$ and Andrey Sorokin ${ }^{1}$
}

'Department of Medicine, ${ }^{2}$ Department of Physiology, and ${ }^{3}$ Department of Pharmacology, Medical College of Wisconsin (MCW), Milwaukee, Wisconsin, USA.

\begin{abstract}
Renal preglomerular arterioles regulate vascular tone to ensure a large pressure gradient over short distances, a function that is extremely important for maintaining renal microcirculation. Regulation of renal microvascular tone is impaired in salt-sensitive (SS) hypertension-induced nephropathy, but the molecular mechanisms contributing to this impairment remain elusive. Here, we assessed the contribution of the SH2 adaptor protein p66Shc (encoded by Shc1) in regulating renal vascular tone and the development of renal vascular dysfunction associated with hypertension-induced nephropathy. We generated a panel of mutant rat strains in which specific modifications of Shc1 were introduced into the Dahl SS rats. In SS rats, overexpression of p66Shc was linked to increased renal damage. Conversely, deletion of p66Shc from these rats restored the myogenic responsiveness of renal preglomerular arterioles ex vivo and promoted cellular contraction in primary vascular smooth muscle cells (SMCs) that were isolated from renal vessels. In primary SMCs, p66Shc restricted the activation of transient receptor potential cation channels to attenuate cytosolic $\mathrm{Ca}^{2+}$ influx, implicating a mechanism by which overexpression of p66Shc impairs renal vascular reactivity. These results establish the adaptor protein p66Shc as a regulator of renal vascular tone and a driver of impaired renal vascular function in hypertension-induced nephropathy.
\end{abstract}

\section{Introduction}

One of the major complications of hypertension is nephropathy, which is associated with impaired renal vascular responsiveness and structural changes (1). The microvascular injuries are detected in the majority of patients with hypertension (2). Even though microvascular injury is among causes of diseases leading to end-stage renal disease, the pathophysiological mechanisms mediating renal microvascular dysfunction remain unknown.

The Src homology 2 domain-containing adaptor protein p66Shc, a longevity-associated product of the Shc1 gene (3), is implicated in the pathogenesis of age-related diseases due to its ability to mediate the cellular sensitivity to oxidative stress (4). Excessive signaling via p66Shc is likely to be one of the mechanisms of age-related pathologies (5). Endothelin-1 (ET-1), a vasoactive peptide that plays an important role in age- and hypertension-related kidney diseases (6), induces the phosphorylation of p66Shc Ser36 residue, which is essential for p66Shc in promoting age-related renal diseases $(3,7)$.

Dahl salt-sensitive (SS) rats, being SS when fed a high-salt diet, exhibit many traits associated with SS hypertension in humans (8). SS rats have become a regularly used model for the study of SS hypertension and accompanying cardiovascular disorders (9-12).

Here, using a zinc finger nuclease-based (ZFN-based) technology for targeted editing of the rat $S h c 1$ gene in SS rats, we estab-

Conflict of interest: The authors have declared that no conflict of interest exists. Submitted: April 8, 2014; Accepted: April 19, 2016.

Reference information: J Clin Invest. 2016;126(7):2533-2546. doi:10.1172/JCI75079. lished that $\mathrm{p} 66 \mathrm{Sh}$ regulates renal vascular tone in isolated preglomerular arterioles and promotes renal vascular dysfunction. The mechanism of action of p66Shc is linked to inhibition of transient receptor potential canonical (TRPC) channel activity, which limits the increase in intracellular $\mathrm{Ca}^{2+}$-induced activation of calciumdependent intracellular signaling pathways and impairs vascular responses. Our data establish a role for the p66Shc in regulation of renal vascular tone and promoting renal vascular dysfunction in hypertension-induced nephropathy.

\section{Results}

Shc proteins are overexpressed in renal tissues from rats with hypertension-induced nephropathy. Consomic rat strains offer unsurpassed inbred controls, allowing physiological comparisons with the parental strains to determine the effect of substitution of a specific chromosome on a disease-susceptible genomic background (13). In the present study, replacement of rat chromosome 2 of SS rats with a corresponding chromosome from the salt-resistant brown Norway $(\mathrm{BN})$ rats (hereafter referred to as SS/BN2) increased survival rate and protected the animals from the development of hypertension-induced renal injury when the rats were fed a $1 \%$ salt diet (Figure 1, A and B). Under these conditions, all consomic SS/BN2 rats survived, whereas less than $25 \%$ of the SS rats survived to 34 weeks of age (31 weeks of $1 \%$ salt diet). Increased survival rate in SS/BN2 versus SS rats was associated with a lesser degree of thickening glomerular and tubular basement membranes and lesser expansion of the mesangial matrix (Figure 1B). This difference in the degree of renal damage 
A

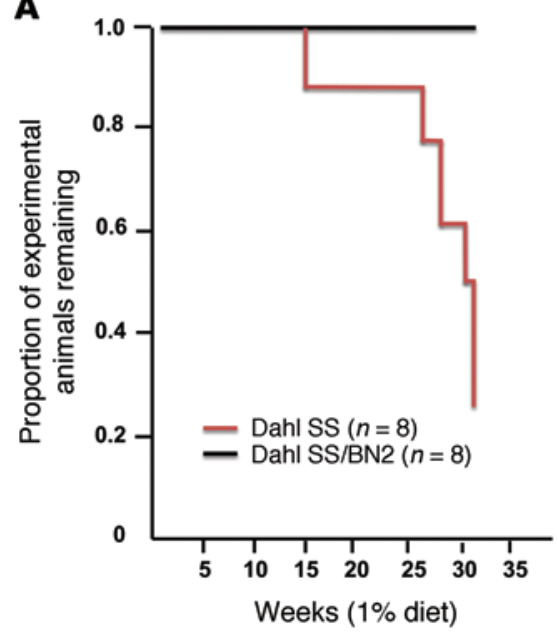

B

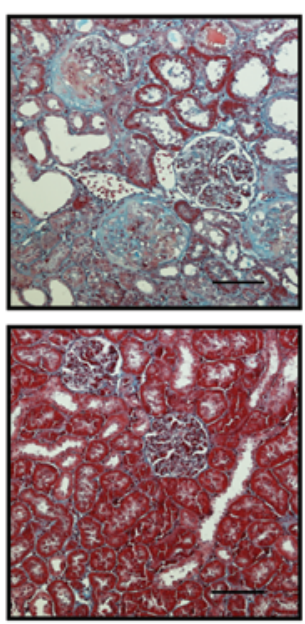

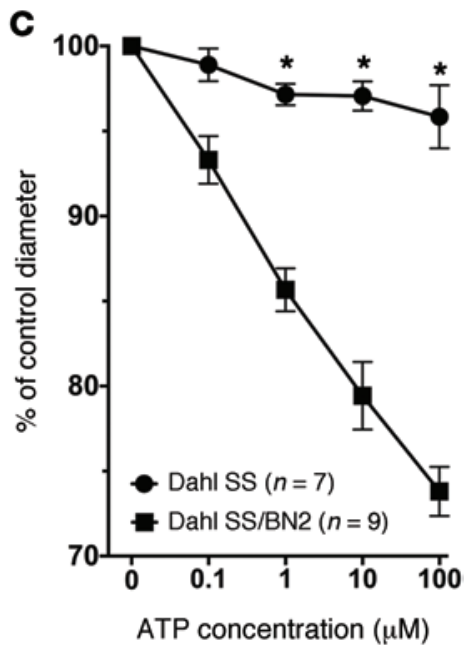

Figure 1. Dahl SS and SS/BN2 consomic rats respond differently to a $\mathbf{1 \%}$ salt diet. (A) Survival of male SS and SS/BN2 consomic rats when fed a $1 \%$ salt diet. (B) Light microscopy of renal cortical sections after Masson's trichrome staining demonstrates the development of renal pathologies in age-matched SS (upper panel) and SS/BN2 (lower panel) rats. Rats were 34-week-old males. Scale bars: $100 \mu$ m. (C) Renal microvascular responses to purinergic activation are impaired in SS rats kept on a 1\% salt diet. Responses of interlobular renal arterioles to ATP were compared in SS rats and SS/ BN2 rats fed a 1\% salt diet. Rats were 15- to 19-week-old males. Statistical comparisons within each series were made using 1-way ANOVA for repeated measures combined with the Newman-Keuls multiple range test. Comparisons between groups were made using 1-way ANOVA with the NewmanKeuls multiple range test. A probability value of $P<0.05$ was considered statistically significant. All data are reported as mean \pm SEM.

could be explained by affected renal hemodynamics in SS rats. Autoregulatory control of preglomerular resistance is an essential component of normal renal hemodynamics (14). It has been previously demonstrated that afferent arteriolar autoregulatory behavior is impaired in Ang II-dependent hypertension (15) and tubuloglomerular feedback signals are coupled to autoregulatory preglomerular vasoconstriction through ATP-mediated activation of P2X1 receptors $(16,17)$. We evaluated the renal microvascular response to purinergic receptor activation in SS rats kept on a $1 \%$ salt diet. The vasoconstrictor responses of renal interlobular arteries to ATP were greatly attenuated in SS rats fed a $1 \%$ salt diet compared with SS/BN2 (Figure 1C) or normotensive Wistar Kyoto (WKY) rats (fed the same diet) (Supplemental Figure 1; supplemental material available online with this article; doi:10.1172/JCI75079DS1), whereas renal interlobular constriction in response to the thromboxane mimetic U46619 (10 nM) was unaltered. Renal interlobular arteries of SS rats treated with U46619 constricted to $71.6 \% \pm 5.4 \%$ of control diameter $(n=5)$, while arteries of SS/BN2 rats responded with constriction to $69.1 \% \pm 3.9 \%$ of control diameter $(n=6)$. Thus, impaired vasoconstrictive response and vascular dysfunction could contribute to renal damage in SS rats kept on a $1 \%$ salt diet, and genetic determinants located on rat chromosome 2 could play a role in the sensitization of SS rats to hypertension-induced microvascular dysfunction. Responses to ATP observed in SS/BN2 rats were greater than previously reported for Sprague Dawley rats (18), probably due to differences in experimental protocols (chronic exposure to $1 \%$ salt diet in the current study) in genetic background and in rat strains' salt sensitivity.

p66Shc is encoded by the gene Shc1, which is located on rat chromosome 2. Using Western blot (WB) analysis, we demonstrated that the level of expression of 3 Shc isoforms, includ- ing p66Shc, in renal tissues was strikingly higher in SS rats when compared with SS/BN2 rats under these experimental conditions (Figure 2A). As revealed by immunohistochemical analysis with antibodies specific to the p66 isoform of Shc, p66Shc expression in renal tissues from rats with hypertensioninduced nephropathy was particularly enhanced in vascular SMCs of renal interlobar artery from SS rats compared with salt-resistant SS/BN2 rats (Figure 2B). WB analysis of proteins from interlobular arteries confirmed the expression of p66Shc in renal blood vessels (Supplemental Figure 2A). Expression of p66Shc in SS rats was increased in rats fed a $1 \%$ salt diet when compared with rats maintained on a low-salt $(0.4 \%)$ diet (Supplemental Figure 2, A and B). Importantly, p66Shc was overexpressed in SMCs of the afferent arterioles, the microvessels that play a key role in regulation of normal renal hemodynamics, of SS rats fed a 1\% salt diet (Figure 2C). Expression of another tested signaling molecule, MnSOD, in renal medulla was similar in age-matched SS and SS/BN2 rats fed a $1 \%$ salt diet (Figure 2A). Thus, we aimed to evaluate the role of p66Shc in renal microvascular dysfunction observed in hypertensioninduced nephropathy. Expression of Shc isoforms is under the control of alternative promoters (19), and direct sequencing of the $S h c 1$ gene from SS and BN rats revealed a number of SNPs in the $S h c 1$ promoter region that could be the cause of differential expression of p66Shc (Figure 3A). Promoter-specific hypermethylation could be associated with aging and age-related diseases (20). However, our analysis did not reveal differences in methylation patterns of the p66Shc promoter between SS and SS/BN2 rats (Figure 3B).

p66Shc KO restored renal microvascular responses and mitigated glomerular damage in hypertensive rats. Whereas the $\mathrm{KO}$ of all $3 \mathrm{Shc}$ isoforms results in abnormal heart development and 
A

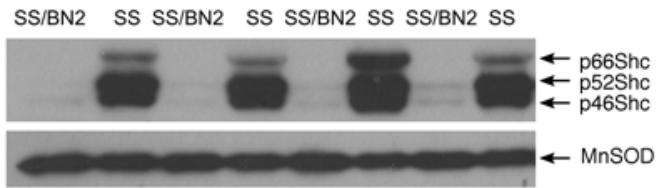

B
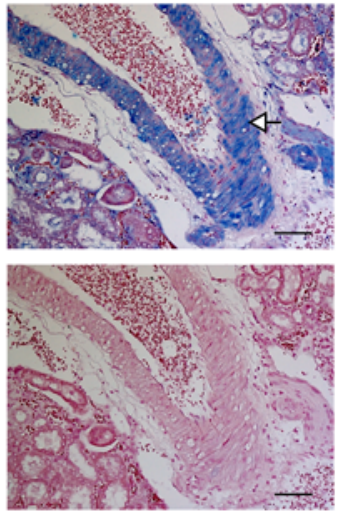

C

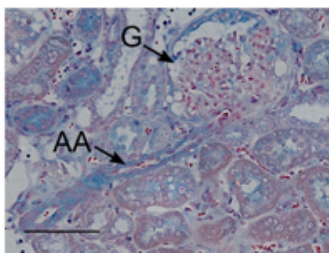

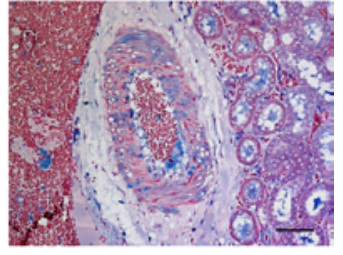
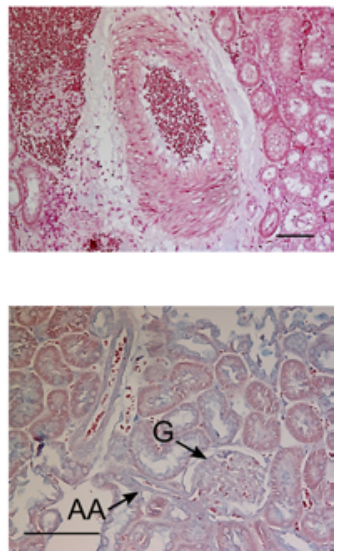

$100 \%$ lethality of mouse embryos, mice lacking only p 66 Shc are viable (21). To address directly the role of p66Shc in hypertension-induced nephropathy of the SS rat, we generated several unique p66Shc mutant strains on the genetic background of SS rats using CompoZr ZFNs (22) targeting exon 2 of the $S h c 1$ gene. Injection of mRNA-encoding ZFNs into SS embryos generated 2 mutant strains with frameshift mutations (termed M1 and M4) (Figure 4A). WB analysis of rat renal tissues confirmed that the M1 and M4 strains were both KOs of the p66Shc isoform, whereas the p46Shc and p52Shc isoforms were not altered (Figure 4B). To generate SS rats that express p66Shc with a mutated phosphorylation site, the template plasmid encoding a Ser36 to Ala36 (Ser36Ala) substitution ( $\mathrm{T}>\mathrm{G}$ transversion) was coinjected in 1-cell embryos along with ZFNs targeting exon 2 of the $S h c 1$ gene in close proximity to the codon encoding p66Shc Ser36 (Figure $4 \mathrm{C})$. Using this innovative in vivo knockin strategy, we generated a single founder animal and established a breeding colony of rats with the Ser36Ala substitution, which was confirmed by sequencing (Figure 4D). Phosphorylation-specific p66Shc antibodies revealed the absence of phosphorylation in ET-1-treated primary cultured smooth muscle cells (SMCs) isolated from renal interlobar arteries of the Ser36Ala knockin strains (Figure $4 \mathrm{E}$ ), verifying that the mutant protein in this strain is no longer a subject of regulation by ET-1.

Glomerular filtration pressure is controlled by appropriate adjustments in preglomerular resistance and might be influenced by p66Shc, which we demonstrated to be overexpressed in vascular SMCs of renal afferent arterioles in the course of hypertension-induced nephropathy (Figure 2C). Since renal

Figure 2. p66Shc is overexpressed in renal tissues from SS rats, but not from salt-resistant SS/BN2 rats. (A) Differential renal expression of Shc proteins in renal medulla of SS and SS/BN2 male rats fed a $1 \%$ salt diet detected by WB. Expression of MnSOD is shown as an equal loading control. Every lane corresponds to a separate animal. (B) Immunohistochemistry shows p66Shc overexpression (blue) in vascular SMCs of the wall of renal interlobar artery in SS rats (upper left), but not in SS/ BN2 rats (upper right). No staining was seen in negative control either in SS (lower left) or SS/BN2 rats (lower right). White arrow shows p66Shc expression in vascular SMCs of a renal vessel. (C) Immunohistochemistry shows p66Shc expression (blue) in afferent arteriole (AA) that is between the interlobular artery and glomerulus (G) of SS (left panel) and SS/BN2 (right panel) rats. Black arrows point to afferent arteriole and glomerulus. Scale bars: $100 \mu \mathrm{m}$.

afferent arterioles are primarily responsible for maintaining pressure gradient between larger vessels and renal capillaries, the impaired ability of afferent arterioles of SS rats to respond to increase in pressure is a marker of renal vascular dysfunction associated with hypertension-induced nephropathy. It has been reported that SS rats have impaired autoregulation of renal blood flow, so the elevated systemic pressure is directly transmitted to the glomerular capillaries and damages the glomeruli (23). We compared microvascular responses of renal afferent preglomerular arterioles to perfused pressure in SS/BN2, 2 strains of p66Shc-KO SS rats, M1 and M4, Ser36Ala mutant rats, and their WT littermates (Figure 5A). Our data strongly suggest that p66Shc KO restored renal microvascular responses to perfused pressure in SS rats fed a 1\% salt diet. As expected, SS/BN2 rats showed prominent autoregulatory response to an increase in perfused pressure. Surprisingly, Ser36Ala mutant rats did not behave as p66Shc KOs M1 and M4, providing what we believe is the first evidence that $\mathrm{p} 66 \mathrm{Shc}$ effects are not restricted to its action in mitochondria (Figure 5A). The ability of p66Shc-KO M1 to restore autoregulation of afferent arterioles was evident even when the perfused pressure was increased to $160 \mathrm{mmHg}$, which corresponds to blood pressure observed in hypertensive SS rats (Supplemental Figure 3). Another marker of autoregulatory activity of renal preglomerular afferent arterioles is the response to ATP. As is the case with renal interlobular arteries (Figure 1C), the vasoconstrictor response of isolated renal preglomerular arterioles to ATP is marginal in SS rats, whereas SS/ BN2 rats demonstrate significant contraction. Using isolated preglomerular afferent arterioles, we demonstrated that p66Shc KO restores vasoconstrictor response to ATP (Figure 5B). p66Shc KO restored contraction of afferent arterioles in response to both ATP (Supplemental Figure 4) and perfused pressure (data not shown) in both male and female rats. Again, Ser36Ala p66Shc mutation was not sufficient to restore autoregulation of afferent arterioles of rats fed a 1\% salt diet (Figure 5B). The effect was specific for renal arterioles, since p66Shc deficiency did not affect the ATP responsiveness of mesenteric arteries (data not shown). Furthermore, we evaluated the effect of p66Shc KO on ET-1-mediated contraction of afferent arterioles. ET-1 is one of the most potent renal vasoconstrictors and is involved in the regulation of renal microvascular function (24). Both SS rats and SS/BN2 rats responded to ET-1 by increased contraction of afferent arterioles (Figure 5C). Accordingly, p66Shc KO was not 
A

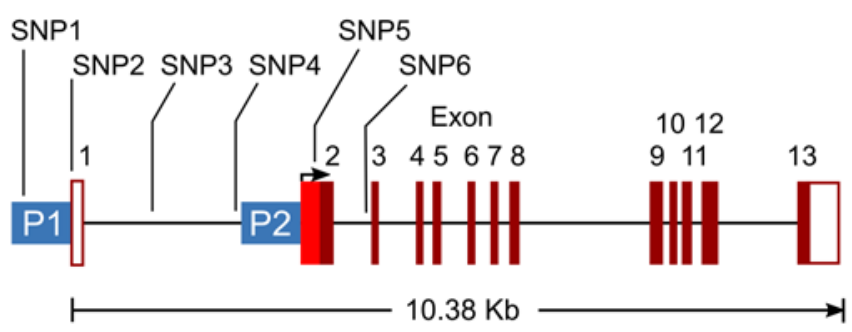

B

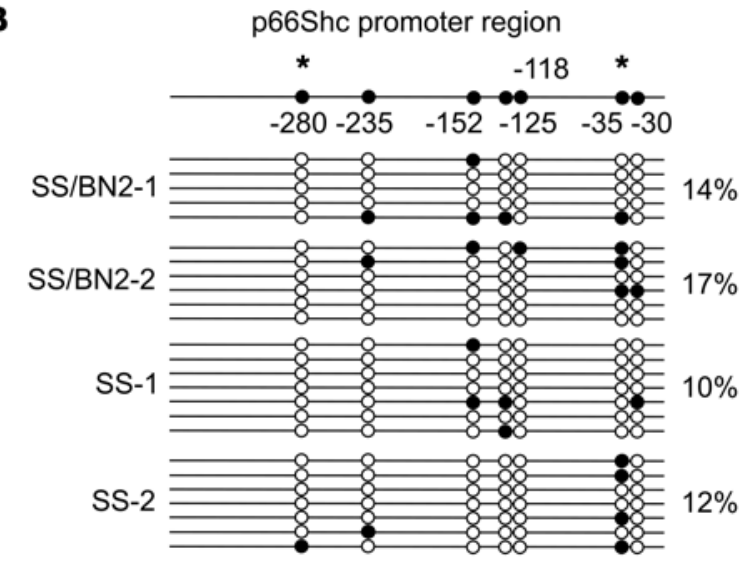

significantly different in response to ET-1 (Figure 5C). Remarkably, afferent arterioles isolated from Ser36Ala rats exhibited an impaired vascular response to ET-1 (Figure 5C).

It was important to verify that our genetically modified rats were not different from SS rats with regard to response to a $1 \%$ salt diet by an increase in blood pressure. Blood pressure measurements were carried out in SS, SS/BN2, M1, M4, and Ser36Ala groups maintained on $1 \%$ salt (Figure 6). Our data demonstrate that SS, SS/BN2, M1, M4, and Ser36Ala rats all have similar increases in mean arterial pressure, and therefore, the differences in blood pressure can be ruled out as a possible cause for differences in autoregulatory responses.

Since glomerular damage is one of the direct consequences of renal vascular dysfunction, we tested to determine whether the restoration of microvascular responses mitigated the glomerular damage in p66Shc-KO mutants. Albumin leakage (albuminuria) resulting from the inability of afferent arterioles to maintain a high vascular tone and, consequently, glomerular damage were higher in WT rats compared with p66Shc-KO rats and SS/BN2 rats after 3 weeks (Figure 7A) and 17 weeks (Figure 7B) maintenance on a 1\% salt diet. Group means of SS/BN2 and M4, but not of Ser36Ala, were different from those of WT SS rats at both time points. The difference between SS rats and either SS/BN2 or p66Shc-KO rats was detectable even when rats were maintained on a $0.4 \%$ salt diet for 17 weeks, due to some degree of albumin leakage detected in SS rats under these conditions (Supplemental Figure 5). Furthermore, we carried out glomerular injury scoring on PAS-stained paraffin sections, adopting the semiquantitative scoring system that was used previously to evaluate the state of glomerulosclerosis in nephrectomized rats (25). The comparison of glomerular damage in p66Shc-KO and WT SS animals revealed the mitigation
Figure 3. SS Shc1 promoter contains SNP1-6. (A) Location of SNP1-6 in Shc1 gene. Positions of alternative promoters for $p 52 / 46$ transcript $(P 1)$ and $\mathrm{p} 66$ transcript (P2) are indicated. (B) Evaluation of Shc1 promoter methylation by the EpiTect Bisulfite Kit. Numbers indicate the position of $\mathrm{CpC}$ sites analyzed relative to the methionine start codon. Horizontal lines represent individual sequenced alleles. Asterisks indicate putative Sp1 transcription factor-binding sites that contain CpC sites. Black and white circles indicate methylated and nonmethylated cytosines, respectively. Percentages indicate the fraction of methylated cytosines found in each rat.

of glomerular damage in the absence of p66Shc (Figure 7, C and D). As in other assays, Ser36Ala mutant rats were not different from parental SS rats in terms of glomerular damage on a $0.4 \%$ salt diet (Figure 7C).

p66Shc mediates effects of ET-1 on cellular contraction, TRPC channel activity, and changes in cytosolic $\mathrm{Ca}^{2+}$ in primary SMCs derived from renal vessels. In order to get insight into mechanisms of p66Shc-mediated impairment of renal microvascular responses, we isolated primary SMCs from renal interlobar arteries of genetically modified rats and WT SS rats. These primary cell cultures were used to test whether the effect of p66Shc KO upon contraction of preglomerular arterioles could be caused by altered ability of SMCs to contract. As confirmed by WB analysis, SMCs from p66Shc-KO rats were characterized by complete absence of the p66Shc isoform, whereas expression of isoforms p52 and p46 was not changed (Figure 8A).

The renal production of ET- 1 is higher in the cortex of rats fed a $1 \%$ salt diet than a $0.4 \%$ salt diet (Supplemental Table 1), controlling for rats' genotype. The effect of a $1 \%$ salt diet on ET-1 production was not significant between various genotype groups (interaction $P=0.48$ ), indicating no differential effect of salt for different genotypes. The level of ET-1 in the renal cortex of SS/BN2 rats fed a $0.4 \%$ salt diet was $7.2 \pm 1.3 \mathrm{pg} / \mathrm{mg}$ protein.

We used collagen gel contraction assay (Figure $8 \mathrm{~B}$ ) to measure cellular contraction and established that p66Shc KO (M1 and M4) significantly increased SMC contraction in response to ET-1, whereas Ser36Ala mutation did not. The Ser36Ala data are in good accordance with our ex vivo observation that renal vascular reactivity is impaired in rats expressing p66Shc and restored by p66Sc KO.

ET-1-mediated regulation of renal microvascular tone is $\mathrm{Ca}^{2+}$ dependent (26). Elevation of intracellular calcium concentration $\left(\left[\mathrm{Ca}^{2+}\right]_{\mathrm{i}}\right)$ is strongly linked to renal microvascular contraction and is crucial for ET-1-induced contraction of SMCs (27). Remarkably, in accordance with these data, we showed that p66Shc attenuates activity of TRPC channels, which mediate influx of $\mathrm{Ca}^{2+}$ in vascular SMCs in response to ET-1. In patchclamp single-channel electrophysiology experiments carried out with primary SMCs, we established that p66Shc KO results in a dramatic increase in TRPC channel activity in response to ET-1 (Figure 9). In contrast, single amino acid mutation of p66Shc Ser36Ala results in a significant decrease of TRPC channel activity when compared with WT cells. KO of p66Shc resulted in an increase of NPo (Figure 9C) and current density (Figure 9D). ET-1 also produced dynamic changes in cytosolic $\mathrm{Ca}^{2+}$ concentration in SMCs derived from p66Shc-KO rats when 


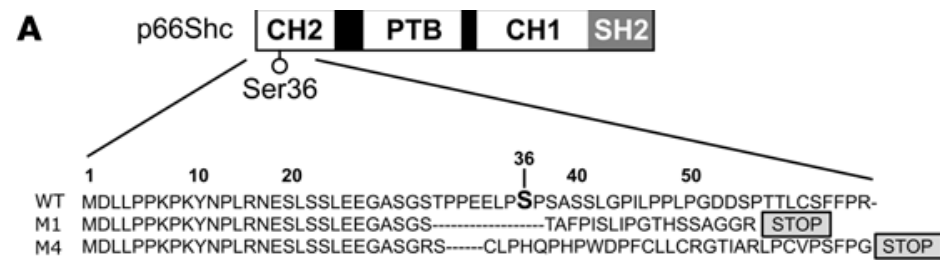

B

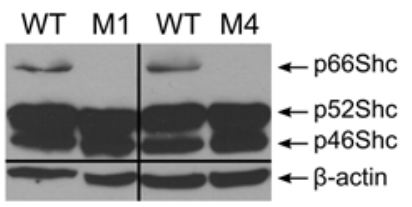

C

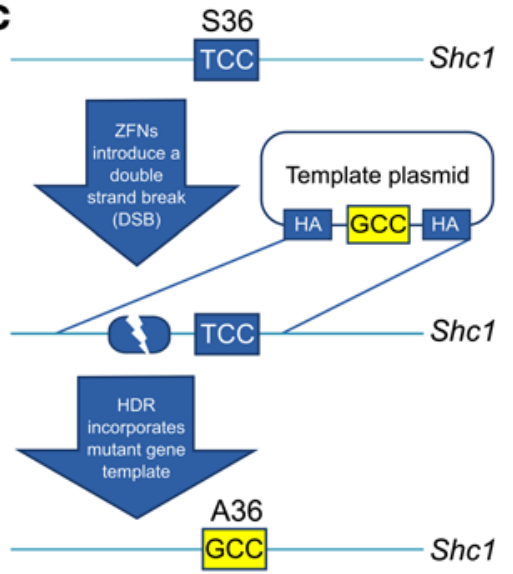

D ZFN Target site Ser36

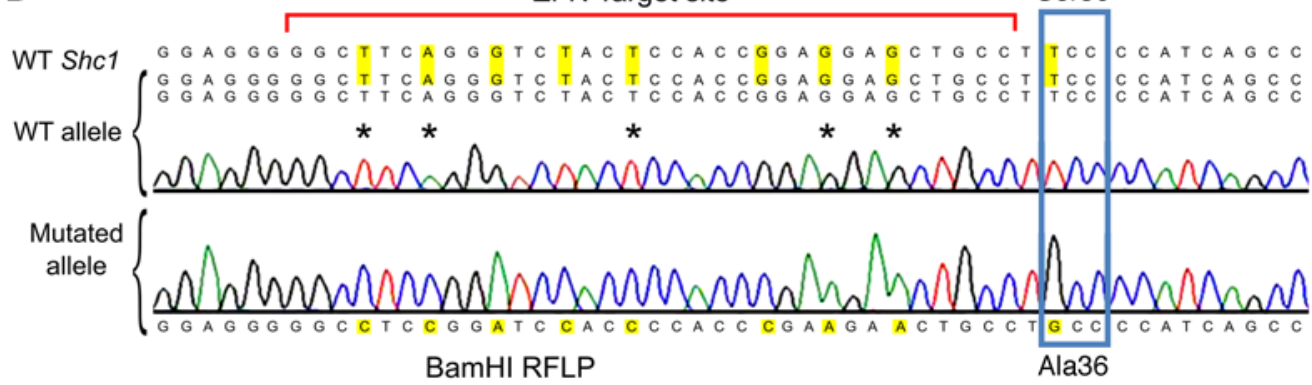

E

\begin{tabular}{|c|c|c|c|c|}
\hline \multicolumn{2}{|c|}{ WT } & \multicolumn{2}{|c|}{ Ser36A } & \multirow[b]{2}{*}{ ET-1 } \\
\hline- & + & - & + & \\
\hline+5 & - & & . & $\begin{array}{l}\text { IP: Shc } \\
\text { IB: pSer36-p66S }\end{array}$ \\
\hline 0 & 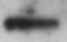 & & 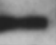 & IB: p66Shc \\
\hline
\end{tabular}

Figure 4. Generation of a panel of mutant rat strains on the genetic background of Dahl SS rats. (A) Schematic diagram of p66Shc protein showing positions of p66Shc domains and regulatory phosphorylation site Ser36 along with the changes in amino acid sequence caused by ZFNs. The newly introduced stop codons are indicated. CH1, collagen homologue 1 region; PTB, phosphotyrosine binding domain. (B) WB revealed deficiency in p66Shc isoform in renal medulla from rats with modified Shc1 gene (M1 and M4), but not in their WT littermates. Blot was probed with antibodies that recognized all 3 Shc isoforms. Equal loading was verified by blotting with anti- $\beta$-actin antibodies. Lanes were run on the same blot, but were noncontiguous. (C) Knockin strategy to generate single amino acid substitution in endogenous rat p66Shc. Ser36Ala substitution strategy used the double-strand break caused by microinjection of ZFNs targeting Shc1 gene to stimulate homology-dependent repair (HDR) and knock in of a Ser36Ala substitution in 1-cell rat embryo. (D) Sanger sequencing confirming introduced Ser36Ala substitution. Raw sequencing data are shown along with sequences of WT and mutated Shc1 alleles. Additional changes were introduced to knock in BamH1 restriction site and to destroy ZFN target site in the edited rat genome. Due to degeneracy of genetic code, these additional changes did not cause amino acid substitutions. (E) WB analysis with phosphospecific antibodies against Ser36 revealed the absence of ET-1-induced Ser36 phosphorylation in SMCs derived from renal vessels of Ser36Ala rats when compared with SMCs derived from their WT littermates. Treatment for 5 minutes with $100 \mathrm{nM}$ ET-1 is indicated by plus signs. Equal expression of p66Shc in these cells was verified by antibodies that recognize p66Shc regardless of its phosphorylation status.

compared with SMCs derived from their WT littermates (Figure 10, A and B). A calcium-sensitive form of Fura 2 (Fura 2-AM) was used to measure changes in the $\left[\mathrm{Ca}^{2+}\right]_{i}$ before and after administration of $100 \mathrm{nM}$ ET-1. In accordance with data related to activation of TRPC channels, the $\left[\mathrm{Ca}^{2+}\right]_{i}$ transient was mark- edly increased in ET-1-treated SMCs isolated from p66Shc KOs M1 and M4 as well as in cells isolated from SS/BN2 rats when compared with SMCs derived from control SS WT rats. Therefore, it appears that Ser36Ala mutation does not result in a similar increase of $\left[\mathrm{Ca}^{2+}\right]_{\mathrm{i}}$ in response to ET-1. ET-1-induced increase 

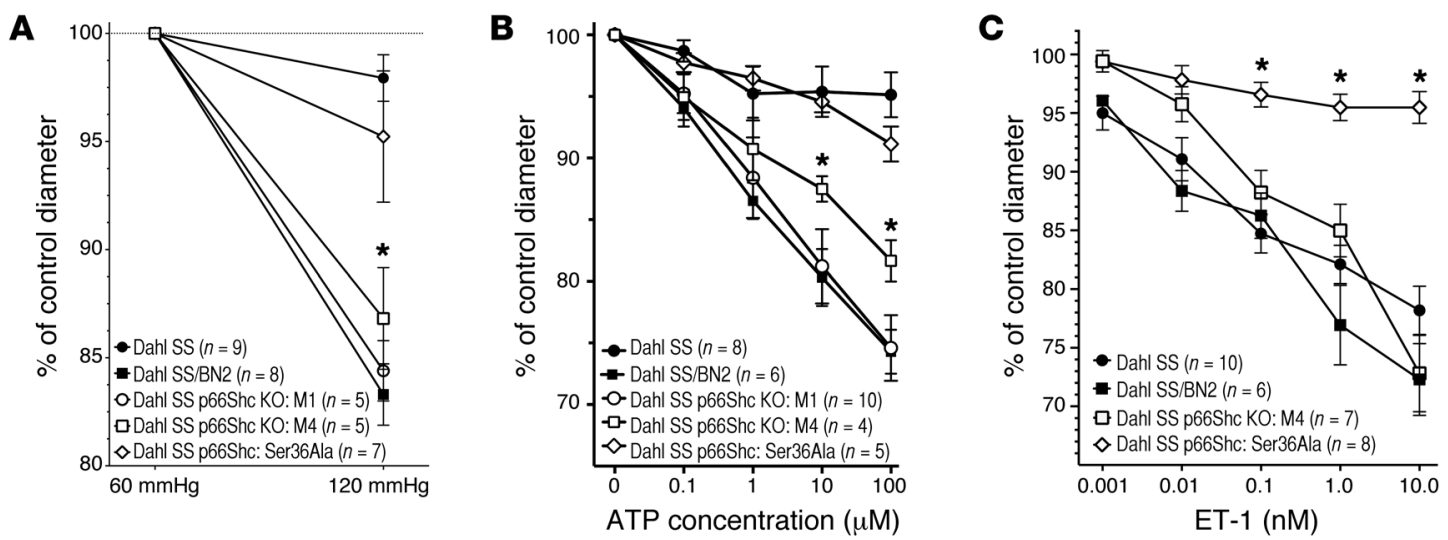

Figure 5. p66Shc KO restored renal microvascular responses in hypertensive rats. (A) p66Shc KO restored renal microvascular responses to perfused pressure in rats. Microvascular responses of renal afferent preglomerular arterioles to perfused pressure were compared in 2 strains of p66Shc KO SS rats, M1 (white circles) and M4 (white squares), their WT littermates (black circles), Ser36Ala mutant (white diamonds), and SS/BN2 (black squares). Shown is normalization to initial afferent arteriolar diameter, which was $19.8 \pm 0.9$ for SS; $21.1 \pm 0.9$ for SS/BN2; $23.4 \pm 1.4$ for M1; $21.7 \pm 0.8$ for M4; and $24.1 \pm 1.7 \mu \mathrm{m}$ for Ser36Ala. (B) p66Shc KO restored renal microvascular responses to purinergic activation in rats. Microvascular responses of renal afferent preglomerular arterioles to ATP were compared in 2 strains of p66Shc KO SS rats, M1 and M4, their WT littermates, and SS/BN2 rats. Shown is normalization to initial afferent arteriolar diameter, which was $24.1 \pm 0.8$ for SS; $20.8 \pm 1.0$ for SS/BN2; $21.4 \pm 1.2$ for M1; $24.1 \pm 2.4$ for M4; and $22.7 \pm 1.4 \mu \mathrm{m}$ for Ser36Ala. (C) ET-1 does not contract isolated renal afferent preglomerular arterioles from Ser36Ala rats. Shown is normalization to initial afferent arteriolar diameter, which was $24.6 \pm 0.8$ for SS; $20.7 \pm 1.8$ for SS/BN2; $23.9 \pm 1.6$ for M4; and $24.5 \pm 1.5$ $\mu \mathrm{m}$ for Ser36Ala. Statistical comparisons within each series were made using 1-way ANOVA for repeated measures combined with the NewmanKeuls multiple-range test. Comparisons between groups were made using 1-way ANOVA with Newman-Keuls multiple range test. ${ }^{*} P<0.05$. All data are reported as the mean \pm SEM.

of $\left[\mathrm{Ca}^{2+}\right]_{\mathrm{i}}$ was dependent on activation of endothelin receptor A (ETRA), since it was inhibited by preincubation of cells with BQ123, an antagonist of ETRA (Figure 10A and Supplemental Videos 1 and 2). We also tested activation of potential $\left[\mathrm{Ca}^{2+}\right]_{i}-$ dependent signaling pathways in renal SMCs isolated either from p66Shc KOs or from WT littermates. We have previously shown that ET-1-mediated activation of calcium-regulated nonreceptor tyrosine kinase Pyk 2 caused an activation of p 38 MAPK (28), which is known to contribute to actin remodeling in SMCs (29). We detected increased activation of p38 MAPK in renal tissues derived from p66Shc KO (Figure 10C) which could be a consequence of more prominent activation of $\left[\mathrm{Ca}^{2+}\right]_{i}$-dependent signaling pathways in the absence of p66Shc.

\section{Discussion}

Our observation that 2 independently generated and genetically distinct $\mathrm{p} 66 \mathrm{Shc}-\mathrm{KO}$ rat mutants demonstrate similar restoration of renal microvascular responses strongly suggests that p66Shc overexpression could cause impaired renal microvascular responses during hypertension.

A reduced ability of the kidney to excrete sodium and water is a major contributor to the development of hypertension and increased blood pressure in response to salt (30). Enhanced renal tubular absorption of sodium and/or excessive renal vasoconstriction impairs pressure natriuresis and leads to hypertension (30). Increased preglomerular vascular resistance can result from increased afferent arteriolar responsiveness to vasoconstrictors such as Ang II and ET-1 (31-34). On the other hand, afferent arteriolar constrictions to increasing renal perfusion pressure or ATP are impaired in hypertension $(16,35)$. The increase in Ang II reactivity in hypertension is not associated with an increase in AT1 receptor expression in renal microvessels $(32,36,37)$. During the established phases of hypertension, enhanced vascular responses to norepinephrine and endothelin are observed $(31,33,34)$. These findings demonstrate that there is enhancement of vascular reactivity to Ang II, ET-1, and norepinephrine, whereas afferent arteriolar constrictions to increasing renal perfusion pressure or ATP are impaired in hypertension. This would suggest that afferent arteriolar responsiveness to Ang II, norepinephrine, and endothelin contributes to increased preglomerular resistance in hypertension. Hence, the afferent unresponsiveness to ATP and responsiveness to vasoconstrictive agents both contribute to hypertension-associated increased preglomerular resistance. Afferent arteriolar unresponsiveness to ATP is an indicator that renal autoregulation is impaired and could contribute to increased glomerular pressure and damage. There is increased afferent arteriolar responsiveness to other vasoconstrictors that could contribute to the hypertension. The rationale for studying p66Shc out of all products of genes on rat chromosome 2 is based on the known ability of p66Shc to mediate cellular sensitivity to oxidative stress and contribute to age-related pathologies (5), which make it a probable causative factor in renal vascular dysfunction (1). In rats deficient in p66Shc, the constriction of afferent arterioles in response to ATP and increased perfused pressure are restored. However, our data with the Ser36Ala mutant, which is not supposed to translocate to mitochondria, a prerequisite for p66Shcmediated increase of mitochondrial ROS production, argue against mitochondrial-based mechanisms of p66Shc inhibition of renal vascular tone in hypertension-induced nephropathy.

In terms of renal afferent arteriole autoregulation, studies of myogenic tone, i.e., responses of renal vessels to increase in pressure, are most important $(38,39)$. The myogenic afferent arteriolar response to high-perfusion pressure is blunted in both SS rats 


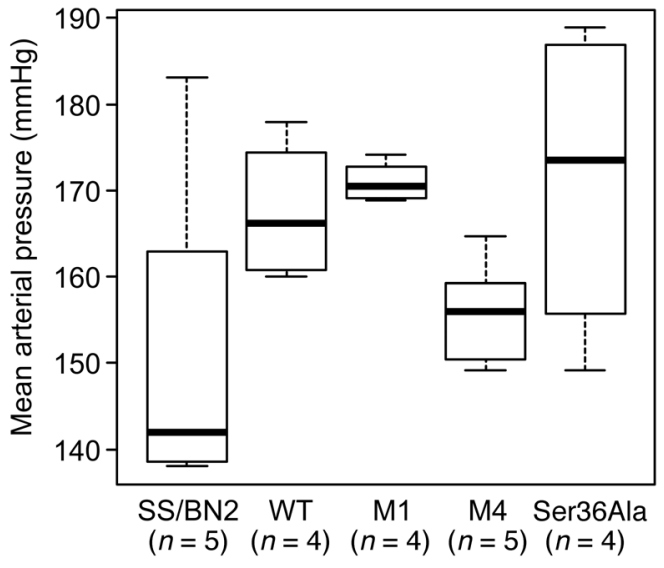

and Goldblatt hypertensive rats (39). Our studies suggest that the altered myogenic response may be associated with p66Shc overexpression and is mediated by signaling via p66Shc.

ET-1 signaling and actions play an essential role for renal biology and pathobiology (40). ET-1 is an important regulator of the renal microcirculation that affects renal function through actions at multiple cell types in kidney, including vascular SMCs (26). We have shown that ET-1 induces p66Shc Ser36 phosphorylation and mediates protein-protein interactions of $\mathrm{p} 66 \mathrm{Shc}$ in renal glomerular cells $(7,41,42)$. Our recent experiments using 2-photon imaging also revealed that treatment of isolated rat renal resistance arteries with ET-1 caused a rapid increase of $\left[\mathrm{Ca}^{2+}\right]_{i}$ in smooth muscle vasculature of these vessels (43).

Members of the TRPC family are abundant in vascular SMCs (44), emerging as important players in the control of smooth muscle function (45). Even though ET-1 causes PKC-dependent activation of TRPC channels (46), these channels also mediate $\mathrm{Ca}^{2+}$ entry in renal afferent arteriolar SMCs in response to noradrenaline and Ang II $(47,48)$. Thus, p66Shc-mediated inhibition of TRPC channels would interfere with signaling triggered by more than one vasoactive agent, resulting in impaired vascular reactivity to a variety of vasoactive agents. ATP plays a significant role in regulation of preglomerular microvasculature (49), and the restoration of ATP-dependent contraction of preglomerular arterioles in rats deficient in $\mathrm{p} 66 \mathrm{Shc}$ argues that upregulation of p66Shc activity may contribute to impaired renal vascular reactivity and the development of hypertension-induced nephropathy. The mechanism of action of p66Shc is linked to inhibition of TRPC channel activity, which limits the increase in $\left[\mathrm{Ca}^{2+}\right]_{i}$-induced activation of calcium-dependent intracellular signaling pathways. Our findings allow us to conclude that p66Shc opposes the activity of TRPC channels, preventing influx of extracellular $\mathrm{Ca}^{2+}$ and inhibiting the vasoconstrictor response in vitro and in vivo. ET-1 and other vasoconstrictors induce phosphorylation of p66Shc Ser36, resulting in intracellular relocalization of p66Shc and activation of TRPC channels.

Even though there is some discrepancy in the consequences of p66Shc KO in SMCs and isolated afferent arterioles with regard to ET-1 stimulation, there is an excellent correspondence between ET-1 action in WT and Ser36Ala p66Shc mutants in both cultured SMCs and afferent arterioles. The possible rea-
Figure 6. All rat strains are characterized by increased mean arterial pressure when maintained on a $1 \%$ salt diet. Mean arterial pressure was evaluated in WT, SS/BN2, and p66Shc-KO rats M1 and M4 as well as Ser36Ala p66Shc mutant rats maintained on a $1 \%$ salt diet for 20 weeks. All animals were males. Means of groups of WT, M1, M4, Ser36Ala, and SS/BN2 were not significantly different from each other. $P=0.1591,1$-way ANOVA.

son for ET-1 being equally active in isolated afferent arterioles from WT and p66Shc-KO rats could be that ET- 1 is an extremely active vasoconstrictor and is capable in the complex context of perfused juxtamedullary nephron preparation of overcoming the p66Shc-mediated impaired renal autoregulation in WT SS rats. Nevertheless, Ser36Ala p66Shc mutation dramatically weakens ET-1 action in both SMCs and afferent arteriole preparations, demonstrating the importance of ET-1 signaling via p66Shc in vitro and in vivo. Primary SMCs offer a simplified experimental model for studying p66Shc action and enabled us to uncover the molecular mechanisms of p66Shc-dependent regulation of renal vascular tone.

In quiescent cells, when p66Shc is not phosphorylated on Ser36, it is localized in the cytoplasm in close proximity to TRPC channels and attenuates their activity (Figure 11A). When Ser36 is phosphorylated (as happens in ET-1-treated cells), it is relocated to mitochondria and p66Shc-mediated inhibition of TRPC channels is alleviated. ET-1 induces activation of TRPC channels and elevation of $\left[\mathrm{Ca}^{2+}\right]_{\mathrm{i}}$ (Figure 11B). In cells expressing Ser36Ala p66Shc mutant, ET-1-triggered signaling cascades are unable to cause Ser36 phosphorylation and, correspondingly, relocation of p66Shc and loss of p66Shc-mediated inhibition of TRPC channels (Figure 11C). Finally, in cells isolated from rat p66Shc KOs where p66Shc is not present to inhibit TRPC channels, ET-1 induces enhanced activation of TRPC channels, increased mobilization of intracellular calcium, and activation of calcium-dependent intracellular signaling pathways (Figure 11D). The exact mechanism of the inhibitory action of p66Shc upon TRPC channels requires further investigation. In accordance with this hypothesized mechanism of p66Shc regulatory action, rat KO of p66Shc restored the myogenic responsiveness of preglomerular renal arterioles ex vivo and enhanced the vasoconstrictor response of primary rat renal arteriolar vascular SMCs. These results establish a role for the adaptor protein p66Shc in the regulation of renal vascular tone and promotion of renal vascular dysfunction.

It must be taken into consideration that vascular SMCs of renal vessels are not the only cells showing the overexpression of p66Shc in rats fed a 1\% salt diet. p66Shc expression also could be detected in glomerular cells (Figure 2C). Glomerular mesangial cells surely are important participants in the regulation of glomerular function, and their abnormal function is often associated with the progression of kidney diseases (50). p66Shc overexpression in mesangial cells could contribute to increased glomerular damage that accompanies hypertension-induced nephropathy, but the regulation of renal vascular tone is likely to be managed by cells of vascular walls of renal vessels.

Finally, our findings raise the possibility that one of the causes of hypertension-induced kidney injury in groups of population with increased risk of developing chronic kidney 
A

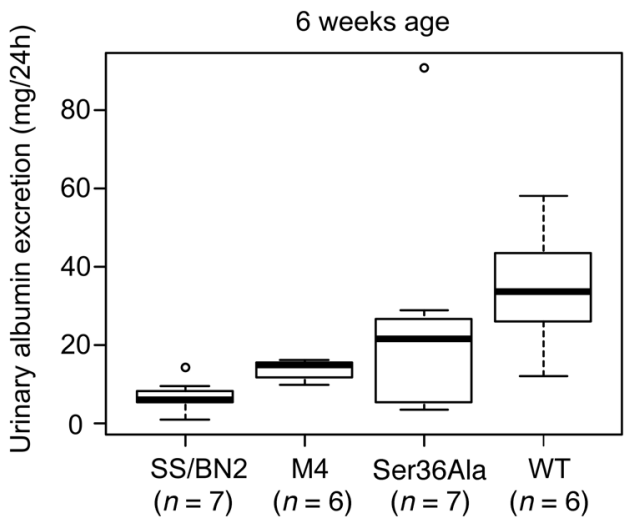

C

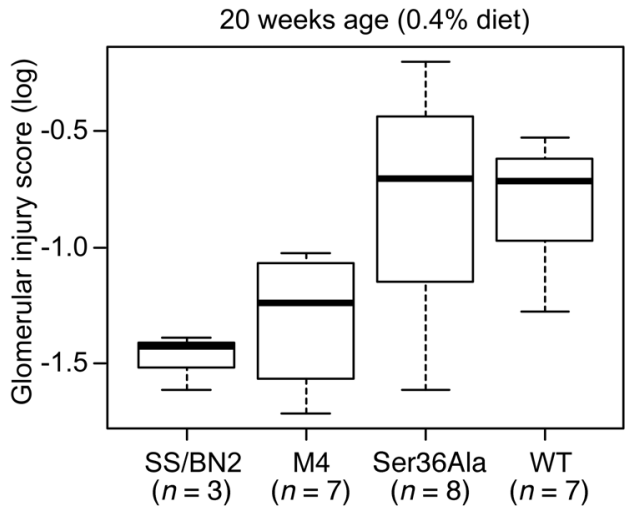

B

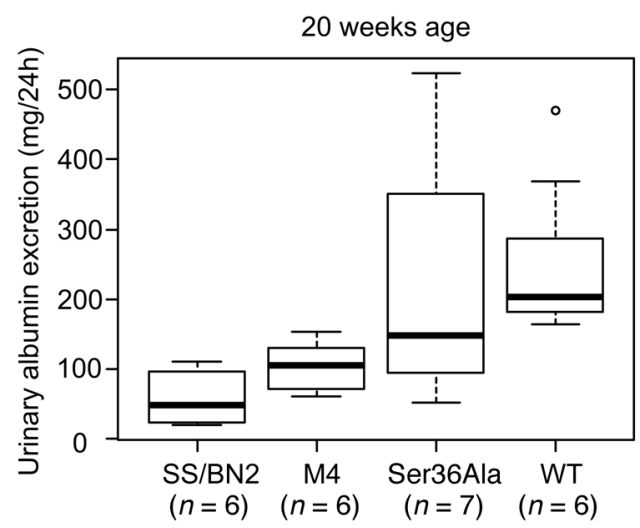

D

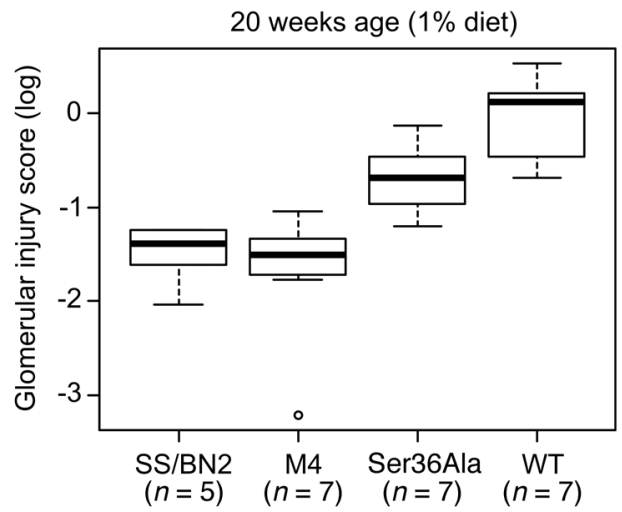

Figure 7. p66Shc K0 mitigated albuminuria and glomerular damage in hypertensive rats. Albuminuria and glomerular damage were compared among WT, SS/BN2, p66Shc KO SS rats (M4), and Ser36Ala p66Shc mutant rats. Comparisons with WT of albuminuria (mg/24 h) in rat groups (M4, Ser36Ala, and SS/BN2) maintained on a $1 \%$ diet at 6 (A) and separately at 20 weeks (B) were performed using the Welch $t$ test, a 2-group $t$ test that accounts for different group variances. Group means of SS/BN2 and M4 were different from WT at both time points $(P<0.05)$. Glomerular damage was compared among WT, SS/BN2, p66Shc KO SS (M4), and Ser36Ala p66Shc mutant rats maintained on either a $0.4 \%$ (C) or a $1 \%$ (D) salt diet (20 weeks of age) using scoring of glomeruli. Comparison was performed using the Welch $t$ tests. M4 group and SS/BN2 groups were significantly different from WT SS group $(P<0.05)$ on a $1 \%$ salt diet. Only SS/BN2 was significantly different from WT on a $0.4 \%$ salt diet.

disease is linked with enhanced signaling via p66Shc. The type of hypertension-induced nephropathy modeled by SS rats is, in many aspects, similar to the one observed in African-Americans $(8,51,52)$. It is of note that, while expression of p66Shc could be driven by ET-1 (41), the increased plasma levels of endothelin have been detected in an African-American population (53). Juxtamedullary afferent arterioles are exposed to high pulsatile pressure and are required to preserve strong vascular tone to maintain the lower glomerular capillary pressure that is necessary for normal function of glomeruli (54). Our finding that p66Shc overexpression in these short vessels that are destined to maintain strong vascular tone attenuates their responsiveness and autoregulatory activity sets the basis for the development of novel therapeutic strategies to combat hypertensioninduced renal vascular dysfunction.

\section{Methods}

Animals. All studies were performed in Dahl SS rats, consomic SS/BN2 rats, BN rats, WKY rats, and genetically modified SS rats. Water was provided ad libitum, and beginning at weaning, animals were maintained on either a low-salt $(0.4 \% \mathrm{NaCl}$, Teklad 7034) diet or a high-salt (1.0\% NaCl, Purina 5001) diet.

Immunohistochemistry. Specific anti-p66Shc antibodies (DiagnoCure) were applied to formalin-fixed paraffin-embedded kidney sections for 1 hour prior to using the mouse-on-rat polymer detection system conjugated with alkaline phosphatase (Biocare Medical), blue alkaline phosphatase substrate as chromagen, and counterstain with Nuclear Fast Red (Vector Laboratories).

Afferent arteriolar responses to vasoactive compounds. Perfused juxtamedullary nephron preparation was used to assess renal microvascular reactivity in rats, as described (55). Briefly, after pentobarbital anesthesia (50 mg/kg, i.p.), the juxtamedullary vasculature was isolated for study and an afferent arteriole was monitored continuously by videomicroscopy. After control diameter measurements, responses to ATP (0.01-100 $\mu \mathrm{mol} / \mathrm{l})$ or ET-1 (0.001-10 nmol/l) were determined in afferent arterioles. Afferent arteriolar diameters were also assessed in response to increasing perfusion pressure from 60 to 120 and from 80 to 120 and $160 \mathrm{mmHg}$ ( 5 minutes each). Arteriolar diameters were recorded at 20-second intervals, and the steady-state diameters were calculated from the average of all diameter measurements obtained 
A
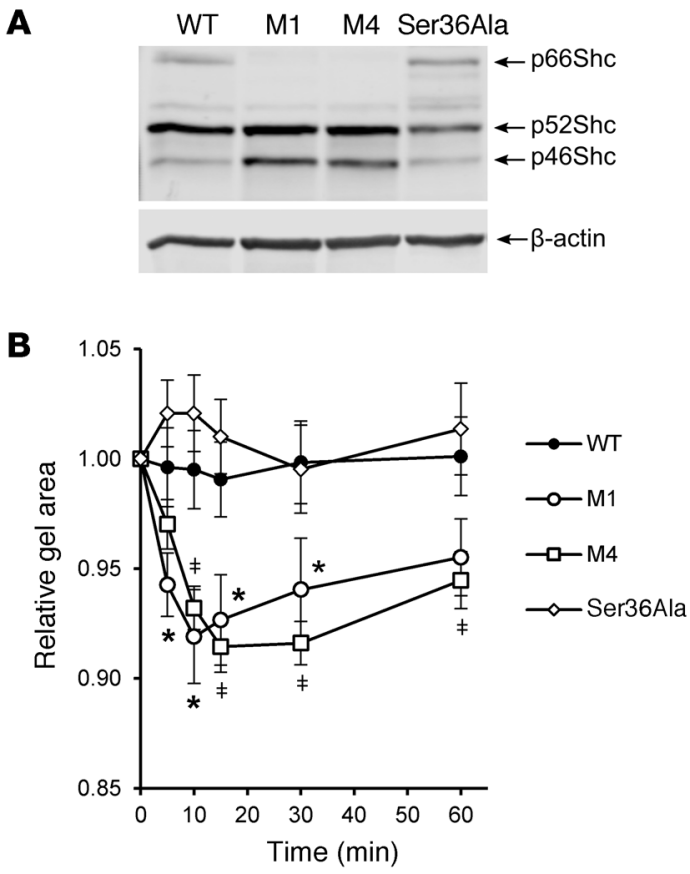

during the final 2 minutes of each 5-minute treatment period. All afferent arteriolar responses to ATP, ET-1, and perfused pressure were measured in animals 13 to 19 weeks old (10 to 16 weeks of $1 \%$ salt diet). Some difference in age of tested animals reflects the difficulty of analyzing more than 2 rats per day.

Measurement of urinary albumin excretion. At 6 and 20 weeks of age, the rats were housed in metabolic caging during a 16- to 24-hour overnight urine collection. Water and food were provided ad libitum. Urine output was determined by volume, taking care to account for debris, and samples were stored at $-80^{\circ} \mathrm{C}$ until assayed. Urinary albumin was quantified with Albumin Blue 580 dye (Molecular Probes) using a fluorescent plate reader (FL600, Bio-Tek).

Analysis of glomerular injury. At the end of the treatment period, rats were euthanized by $\mathrm{CO}_{2}$ inhalation and the right kidney was removed, sectioned longitudinally, and fixed by immersion in $10 \%$ zinc formalin for 3 days. Formalin was replaced with $70 \%$ ethanol until sections were embedded in paraffin blocks for further processing. Four-micron kidney sections were stained with either Masson's trichrome or PAS reagent and counterstained with Harris hematoxylin. Images were collected by a Nanozoomer whole-slide scanner (Hamamatsu Photonics). The semiquantitative glomerular score of each animal was determined in a blinded manner by 2 investigators and derived as the mean of 50 glomeruli. The degree of glomerular injury was determined using a scale from 0 to 4 as described by Adamczak et al. (25). The glomerular score for individual glomeruli was as follows: grade 0 , normal glomerulus; grade 1 , beginning of mesangial expansion and/or thickening of basement membrane; grade 2, mild and moderate segmental sclerosis involving less than half of the glomerular tuft; grade 3, diffuse glomerular sclerosis involving more than half of the tuft; and grade 4, diffuse glomerulosclerosis with total tuft obliteration and collapse.

Promoter analysis and methylation measurements. The complete promoter region of the $S h c 1$ gene locus encompassing SNPs 1-5 was amplified by PCR from tail genomic DNA obtained from BN and SS
Figure 8. Increased ET-1-induced contraction of primary SMCs derived from renal vessels of $\mathbf{p 6 6 S h c - K O}$ rats. (A) SMCs isolated from interlobar artery of M1, M4, Ser36Ala, and their WT littermates expressed Shc isoforms as detected by WB analysis. Positions of Shc isoforms are indicated. (B) KO of p66Shc increased ability of renal primary SMCs to contract collagen matrix to a higher degree in response to ET-1 (100 nM). Shown is average of 4 independent experiments. Two-factor ANOVA for repeated measures followed by planned comparison $t$ tests was performed. M4 and M1 were significantly different from WT and Ser36Ala. ${ }^{\ddagger} P<0.05$, M4; ${ }^{*} P<0.05, M 1$. Ser36Ala was not significantly different from WT. Data are reported as mean \pm SEM.

rats. Bisulfite modification of isolated DNA was performed using the EpiTect Bisulfite Kit (QIAGEN). Conversion rates exceeded 95\%. Both strands of converted DNA were amplified with PCR primers specific to the p66Shc promoter region $(-384$ to -9$)$. Products were cloned into TOPO-TA pCR2.1 (Invitrogen). Individual colonies were grown, and isolated plasmids were sequenced.

Isolation and culture of renal interlobar arterial SMCs. Six- to eight-week-old male rats were euthanized by $\mathrm{CO}_{2}$ inhalation. From 2 to 4 kidneys were extracted into dissociation buffer (3\%-6\% BSA/ high-glucose DMEM with $10 \mathrm{mM}$ HEPES, pH 7.4, and antibiotic/ antimycotic cocktail) on ice for each preparation. The outer capsule was removed, and the kidneys were longitudinally bisected to remove the medulla, exposing the interlobar arteries. Arterial segments were dissected and put into fresh, ice-cold dissociation buffer. Arteries were transferred to another vial and incubated for 20 minutes at $37^{\circ} \mathrm{C}$ in $1 \mathrm{ml}$ solution of dissociation buffer with 0.5 $\mathrm{mg} / \mathrm{ml}$ dithiothreitol and $60 \mathrm{U} / \mathrm{ml}$ activated papain. The supernatant was removed and replaced with $0.5 \mathrm{ml}$ of dissociation solution containing $240 \mathrm{U} / \mathrm{ml}$ collagenase type II, $15 \mathrm{U} / \mathrm{ml}$ elastase, and 0.1 $\mathrm{mg} / \mathrm{ml}$ trypsin inhibitor and incubated at $37^{\circ} \mathrm{C}$ for no longer than 30 minutes. All enzymes used for isolation of SMCs were from Worthington Biochemical Corp. Upon complete dispersion of arterial segments, $2 \mathrm{ml}$ of growth medium (dissociation buffer with $10 \%$ fetal bovine serum) was added and the preparation centrifuged at $1,000 \mathrm{~g}$ for 5 minutes at $4^{\circ} \mathrm{C}$. Single vascular SMCs were resuspended in growth medium and cultured in 6-well plates at $37^{\circ} \mathrm{C}, 5 \% \mathrm{CO}_{2}$. Cultured cells displayed the typical hill-and-valley growth pattern and stained positive for smooth muscle $\alpha$-actin.

Targeted Shc gene destruction using engineered ZFNs. p66Shc rat KOs were generated using engineered ZFNs (56). One-cell embryos were extracted from SS rats, and mRNA encoding 2 engineered ZFNs targeting a portion of the $S h c 1$ gene encoding the p66Shc isoform was injected into the embryo. In order to knock in a Ser36Ala substitution, we used the double-strand break caused by microinjection of ZFNs targeting the Shc1 gene to stimulate homology recombination with the coinjected template plasmid containing the desired mutation.

Collagen gel contraction assay. Twenty-four-well plates were coated with $1 \%$ BSA/PBS overnight, then washed with PBS. Collagen gels were generated by mixing trypsin-harvested SMCs $\left(4 \times 10^{5}\right.$ cells $/ \mathrm{ml}$; passage 9-17) in $0.1 \%$ BSA/DMEM with rat tail collagen I $(1.2 \mathrm{mg} / \mathrm{ml}$; BD Biosciences) and plating $250 \mu \mathrm{l}$ of the resulting cell suspension (50,000 cells) in triplicate. After polymerization for 1 hour at $37^{\circ} \mathrm{C}$, gels were gently detached from the well in $400 \mu \mathrm{l}$ of $0.1 \% \mathrm{BSA} / \mathrm{DMEM}$. Floating gels containing SMCs underwent spontaneous contraction and were allowed 24 hours of equilibration before treatment. Images were tak- 
A

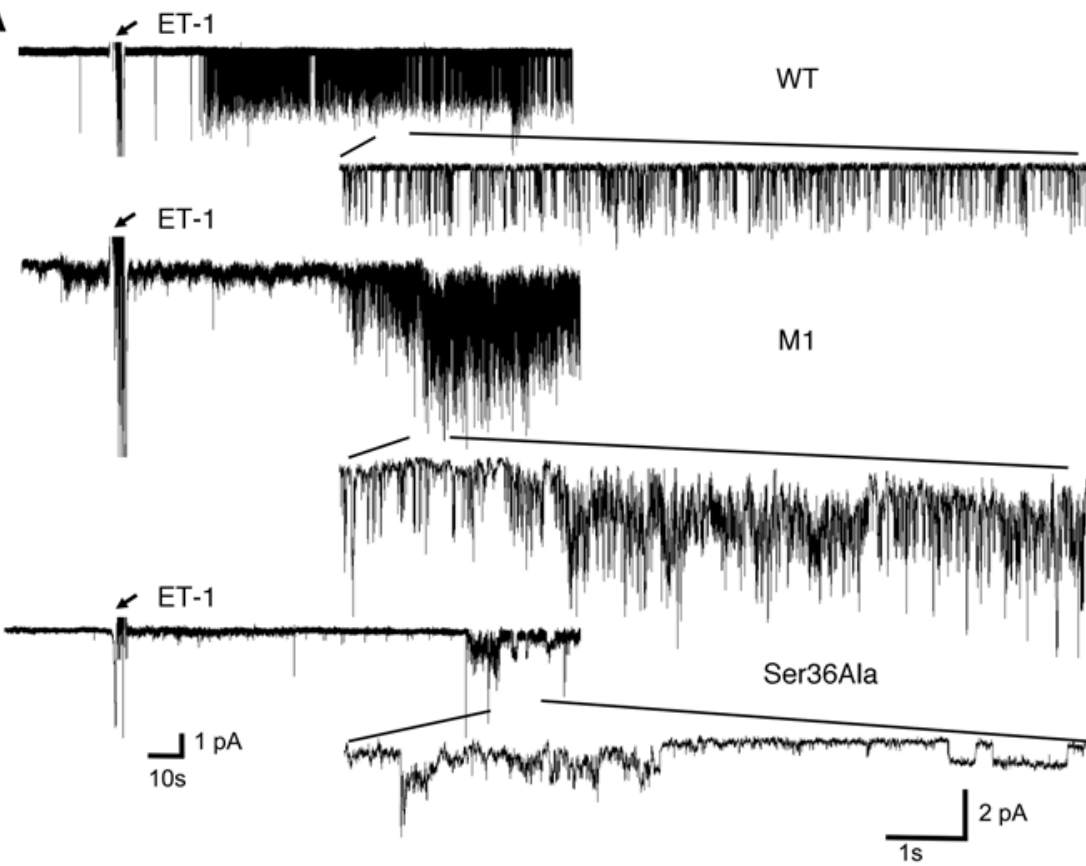

B

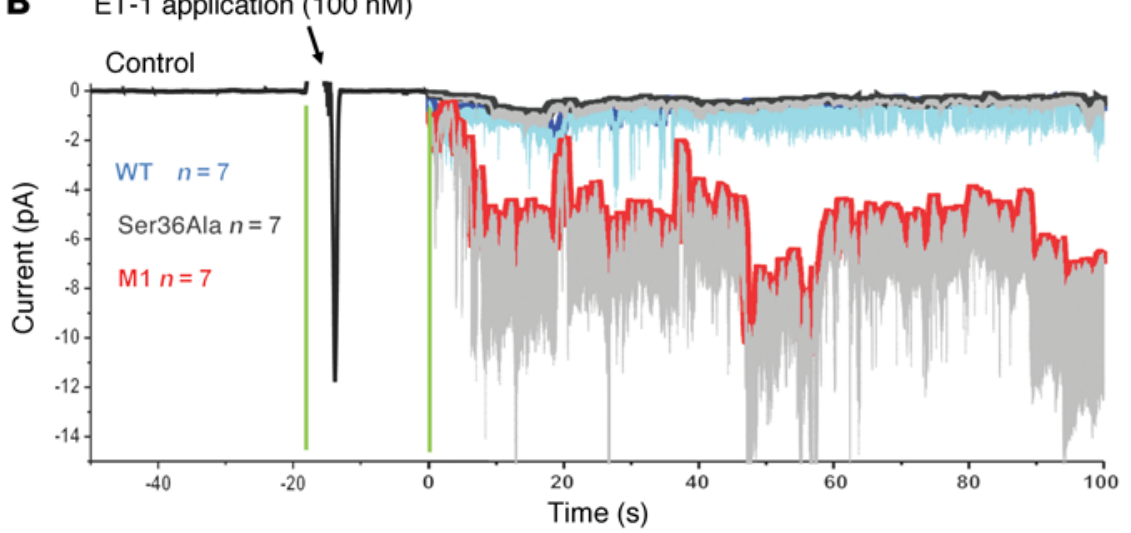

C

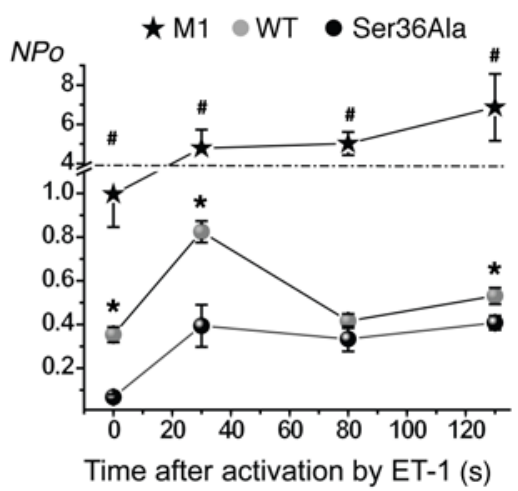

D

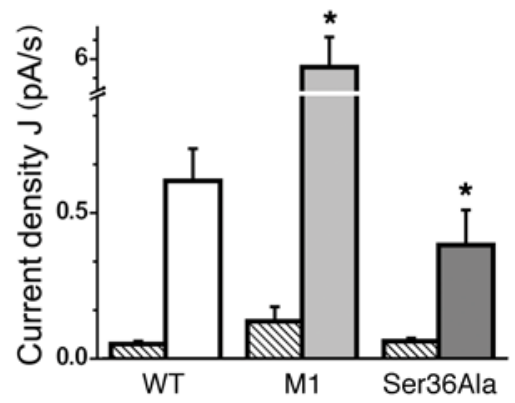

Figure 9. p66Shc actions mediate effect of ET-1 on TRPC channel activity. (A) Representative single-channel traces on vascular SMCs before and after ET-1 treatment in cells derived from WT, M1, and Ser36Ala rats. All traces were recorded at $-80 \mathrm{mV}$ holding potential. Insets show 10 -second single-channel recording intervals. (B) Point-wise comparison of means of single-channel amplitude changes after ET-1 application. Each curve represents the sum of 7 independent experiments. (C) ET-1 (100 nM) induces changes in TRPC channel activity (NPo). M1 is significantly different from WT or Ser36Ala ( $P<0.05)$. WT significance difference with Ser36Ala is also shown ( ${ }^{*} P<0.05$ ). (D) Changes in current density J, $\mathrm{pA} / \mathrm{s}$ calculated as total current integral ratio to the first 100 seconds after activation start point. Ser36Ala significantly differs from WT. M1 significant increase versus WT or Ser36Ala is indicated. ${ }^{*} P<0.05$. Striped bars represent control value before ET-1 application. All summarized data are reported as mean $\pm \mathrm{SEM}$. Data from before and after treatment within the same experiment were compared using the paired $t$ test. Data from different experiments were compared with a Student's $t$ test (2 tailed) or 1-way ANOVA as appropriate.

en immediately before and after treatment with $400 \mu \mathrm{l}$ of $0.1 \%$ BSA/ DMEM with or without 100 nM ET-1. Gel areas were measured using Image $(\mathrm{NIH})$ software at each time point. Relative contraction at each time point was defined as the ratio of percentage change in the area of ET-1-treated gels relative to time equal to 0 to the average percentage change in the area of 3 replicate control gels for each cell line in each experiment. A value of less than 1 denotes a greater degree of contraction of ET-1-treated cells compared with control.

Electrophysiology. Single-channel currents were measured using cell-attached technique with Axopatch 200B amplifier (Molecular Devices) at room temperature at a holding potential of $-80 \mathrm{mV}$. Current recordings were acquired at $1 \mathrm{kHz}$ with Digidata $1440 \mathrm{~A}$ (Molecu- lar Devices) and pCLAMP v10.2 software and filtered with an 8-pole, low-pass Bessel filter LPF-8 (Warner Instruments) at $0.2 \mathrm{kHz}$. After a high-resistance seal (over $10 \mathrm{G} \Omega$ ) was obtained, cell-attached recordings were performed immediately. The membrane resistance was monitored regularly to ensure the quality of recording. For measurements of acute effect, only 1 experiment was performed per coverglass to avoid any possibility of examining cells whose properties might have been altered by extended exposure to ET-1. The resistance of the pipette in the corresponding bath medium was 7-12 M $\Omega .5 \mu \mathrm{M}$ nicardipine, $100 \mu \mathrm{M}$ DIDS, and $100 \mu \mathrm{M}$ niflumic acid were included to block voltage-dependent $\mathrm{Ca}^{2+}$ channels and $\mathrm{Ca}^{2+}$-activated and swellactivated $\mathrm{Cl}^{-}$conductances. 
A WT
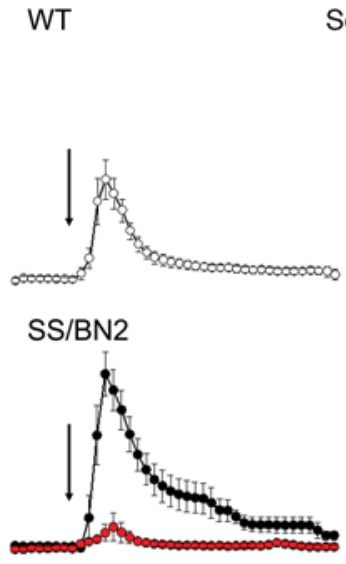

Ser36Ala

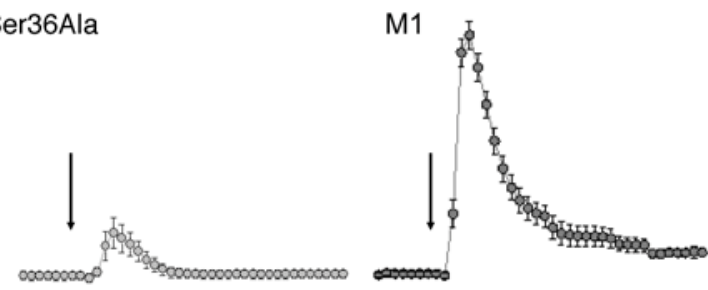

M4

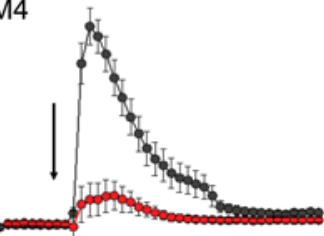

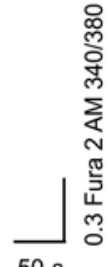

B

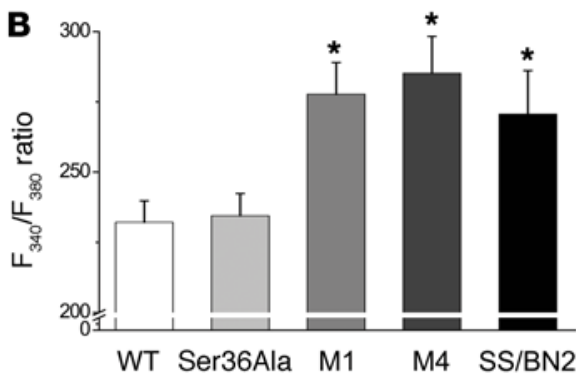

C

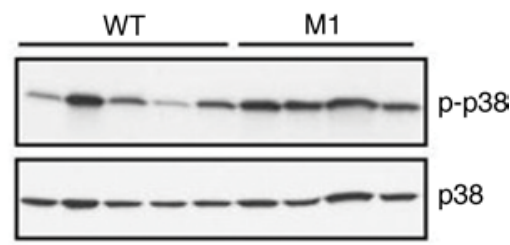

Figure 10. p66Shc actions mediate effect of ET-1 on changes in intracellular $\mathrm{Ca}^{2+}$ in renal SMCs derived from genetically modified rats. (A) ET-1 produced dynamic changes in $\left[\mathrm{Ca}^{2+}\right]_{\mathrm{i}}$ in SMCs. Presented are average time courses of $\left[\mathrm{Ca}^{2+}\right]_{i}$ changes in individual SMCs following exposure to ET-1 (100 nM, arrows) given as relative $\mathrm{F}_{340} / \mathrm{F}_{380}$ ratio. Number of cells analyzed for each strain was as follows: WT $(n=19)$, M1 $(n=51)$, Ser36Ala ( $\left.n=28\right)$, M4 ( $n=16$ in the absence of BQ123 and $n=13$ in the presence of BQ123, marked in red), and SS/BN2 ( $n=11$ in the absence of BQ123 and $n=8$ in the presence of BQ123,

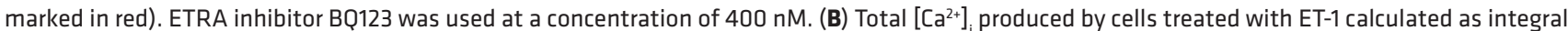
from beginning of activation to the $F_{340} / F_{380}$ ratio level restitution. Comparison was performed using 1-way ANOVA with Bonferroni's correction. M4, M1, and SS/BN2 groups were significantly different from WT and Ser36Ala groups. ${ }^{*} P<0.05$. All summarized data are reported as mean \pm SEM. (C) Increased activation of p38 MAPK in renal tissues derived from p66Shc-KO rats. Renal cortex tissues were lysed and subjected to WB analysis with antibodies that recognize phosphorylated forms of p38 MAPK. Expression levels of p38 MAPKs are also shown. Every lane corresponds to a separate animal. Rats were 26to 28 -week-old males (23-25 weeks on $1 \%$ salt diet).

Fluorescence loading and measurements. The fluorescent $\mathrm{Ca}^{2+}$ indicator Fura 2-AM was used to measure $\left[\mathrm{Ca}^{2+}\right]_{\mathrm{i}}$ changes during $100 \mathrm{nM}$ ET-1 applications. Cell culture was incubated in PSS extracellular solution containing $2 \mu \mathrm{M}$ Fura 2 -AM and $0.05 \%$ pluronic acid for 40 minutes in an incubator in the dark. After incubation, cells were washed with PSS for 15 minutes to remove excess dye. All fluorescent imaging experiments using cultured SMCs were performed in PSS solution. Fluorescence images of changes in the $\left[\mathrm{Ca}^{2+}\right]_{\mathrm{i}}$ level probed with Fura 2-AM were obtained using a Nikon TE-2000U inverted microscope equipped with a 40/1.3 DIC oil immersion objective lens (Nikon S Fluor) and a Zyla sCMOS camera (Andor Technologies). Excitation was provided by a Lambda XL xenon arc lamp at alternating wave lengths, and emission control was achieved using an optical filter changer with integrated shutter/filter wheel driver (Sutter Instruments). Cells were alternately excited at $340 \mathrm{~nm}$ and 380 $\mathrm{nm}$, and the respective 510-nm emissions were acquired every 8 seconds. Changes in $\left[\mathrm{Ca}^{2+}\right]_{i}$ were based on $340 / 380$ ratios.

Extraction and measurement of tissue ET-1. Renal cortical tissue was collected from animals, frozen on dry ice, and stored at $-80^{\circ} \mathrm{C}$. Protein was extracted from approximately $100 \mathrm{mg}$ frozen tissue on ice in $1 \mathrm{ml} 1 \mathrm{M}$ acetic acid with cOmplete Mini protease inhibitor cocktail (Roche) and PMSF with a Potter-Elvehjem homogenizer, followed by two 30-second pulses of sonication. Samples were heated for 10 minutes at $95^{\circ} \mathrm{C}$, placed on ice, and centrifuged at 3000 $g$ for 30 minutes at $4^{\circ} \mathrm{C}$. Supernatants were collected and stored at $-80^{\circ} \mathrm{C}$ until analysis. The $100 \mu \mathrm{l}$ samples were measured for ET-1 content using QuantiGlo ET-1 ELISA (R\&D Systems) according to the manufacturer's instructions, using the supplied ET-1 standard in $1 \mathrm{M}$ acetic acid for standard curve generation. Results were standardized to supernatant total protein using the Pierce BCA Protein Assay Kit (Thermo Fisher Scientific).
Blood pressure measurements. Rats were deeply anesthetized with a mixture of ketamine (75 mg/kg i.p.), xylazine $(10 \mathrm{mg} / \mathrm{kg}$ i.p.), and acepromazine $(2.5 \mathrm{mg} / \mathrm{kg}$ i.p.), with supplemental anesthesia administered as needed. Using aseptic techniques, a telemetry transmitter (model PA-C40, Data Sciences International) for measuring arterial blood pressure was implanted in the carotid artery, with the body of the transmitter implanted s.c. in the back of the animal. Blood pressure was monitored continuously by telemetry, utilizing the implantable transmitter and placing the rat cages upon a receiver/antenna that is part of a commercially available physiological monitoring system. Animals were maintained on warming trays during and following surgery. Analgesics and antibiotics were administered after surgery to control pain and infection. Following a 5- to 7-day recovery after transmitter implantation, arterial blood pressure was continuously monitored for 5 days as we have previously described (57).

Statistics. Statistical methods are described in the corresponding figure legends. The Welch $t$ test, a 2-group $t$ test that accounts for different group variances, was used to compare albuminuria and glomerular damage between groups of rats. Channel openings were analyzed by Clampfit 10.2 software using the single-channel search in the analyze function. Channel activity (NPo), the product of the number of channels (N) and open probability $\left(\mathrm{P}_{\mathrm{o}}\right)$, was used as a measure of channel activity and was calculated using the detection module in pCLAMP software v.10.2. Changes in current density were calculated as total current integral ratio to the first 100 seconds after channel activation by ET-1. Figure preparation and statistical analyses were carried out using MicroCal Origin software 9.0 (MicroCal Software Inc.). Fluorescent images were processed with Image $1.42(\mathrm{NIH})$. Data from before and after treatment within the same experiment were compared using the paired, 2-tailed $t$ test. Comparison was performed using 1-way ANOVA with Bonferroni's 


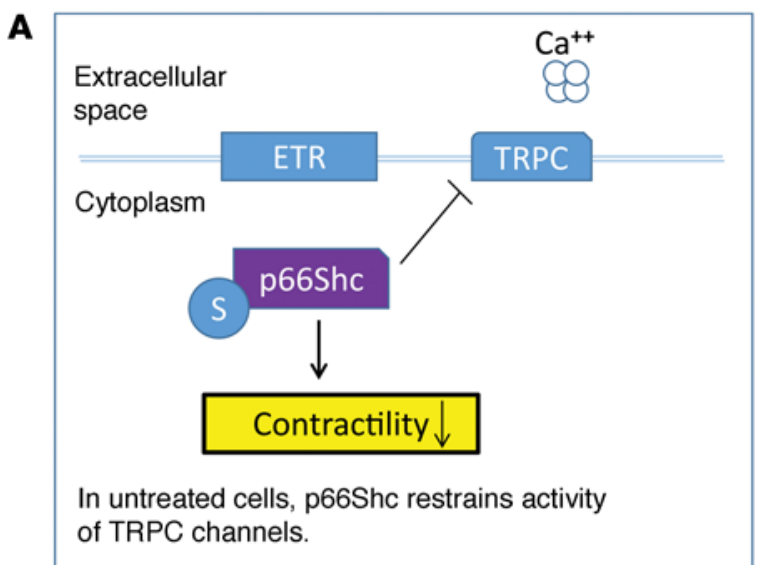

C

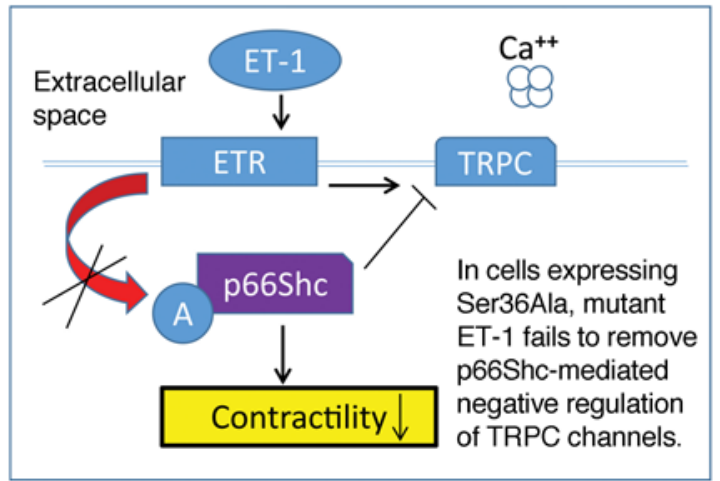

B

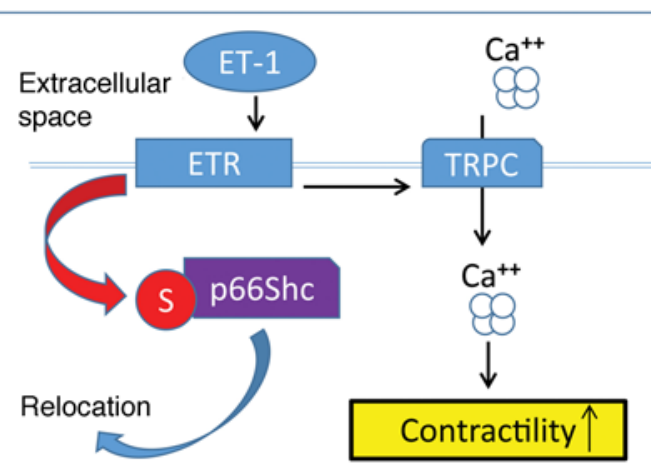

ET-1-mediated Ser36 phosphorylation of p66Shc removes negative regulation of TRPC channels.

D

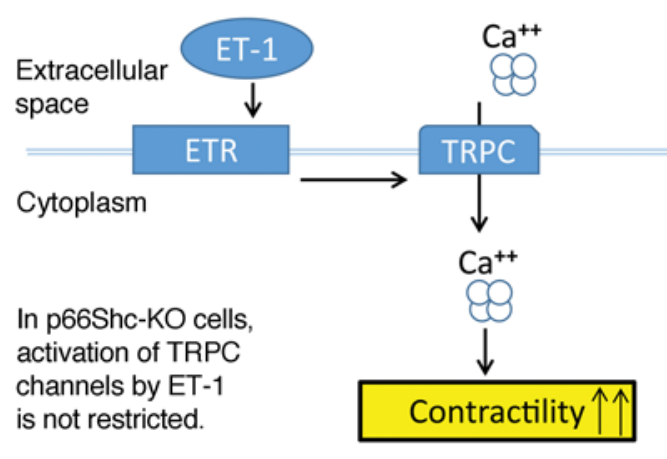

Figure 11. ET-1 signals in SMCs through p66Shc. Schematic of proposed regulation of cellular contraction by p66Shc. (A) In quiescent cells with unoccupied endothelin receptors (ETR), p66Shc is not phosphorylated and restrains activity of TRPC channel. (B) Activation of ET-1 signaling cascade results in Ser36 phosphorylation and intracellular relocation of p66Shc accompanied by activation of TRPC channels. (C) In cells expressing p66Shc with mutated phosphorylation site, the negative regulation of TRPC channels by p66Shc prevails and contraction remains low. ET-1 is unable to disable inhibitory activity of p66Shc. (D) In cells deficient in p66Shc, activation of TRPC channels by ET-1 is dramatically increased due to the absence of the restraining activity of p66Shc.

correction. $P<0.05$ was considered significant. Statistical comparisons of afferent arteriolar dose responses were made using 1-way ANOVA for repeated measures combined with the Newman-Keuls multiple-range test. Comparisons between groups were made using 1-way ANOVA with the Newman-Keuls multiple-range test. Data analysis for SMC collagen matrix contraction was generated using Real Statistics Resource Pack software (release 4.3; copyright 2013-2015, Charles Zaiontz; www.realstatistics.com). All data are reported as mean \pm SEM.

Study approval. Animal use and welfare procedures adhered to the NIH Guide for the Care and Use of Laboratory Animals (National Academies Press. 2011.) and followed protocols reviewed and approved by the MCW Institutional Animal Care and Use Committee.

\section{Author contributions}

BM, OP, VAR, AC, JL, DM, JMW, AMG, and JDI performed the experiments. BM, OP, HJJ, RJR, AWC, A Staruschenko, JDI, and A Sorokin conceived the experimental designs. BM, OP, A Staruschenko, JDI, and A Sorokin analyzed the data and wrote the manuscript.

\section{Acknowledgments}

Specific anti-p66Shc antibodies were provided by DiagnoCure. We thank Marie Barabas (MCW) for help with $\left[\mathrm{Ca}^{2+}\right]_{\mathrm{i}}$ recording, Camille P. Taylor (MCW) for help with albumin measurements, Christine Duris (MCW) for histological preparations, and Sergey Tarima
(MCW) for help with statistical analysis of renal injury. We acknowledge the contribution of the Children's Hospital of Wisconsin Children's Research Institute Histology Core, particularly Christine Duris, Stephanie Wirsbinski, Tatunya Bufford, and Qiuhui Yang, for slide preparation and histological staining. This work was supported by NIH grants DK098159 and CA182114 to A. Sorokin, HL108880 to A. Staruschenko, DK038226 to J.D. Imig, HL116264 to D. Mattson and A.W. Cowley Jr., HL122662 to A. Staruschenko and A.W. Cowley Jr., HL036279 and DK104184 to R.J. Roman, and OD008396 to A.M. Geurts and in part by the National Center for Advancing Translational Sciences, NIH, through grant 8UL1TR000055. A. Chong was supported by NIH grant 1R25 DK098104.

Address correspondence to: Andrey Sorokin, Medical College of Wisconsin, Department of Medicine, 8701 Watertown Plank Road, Milwaukee, Wisconsin 53226, USA. Phone: 414.456.4438; E-mail: Sorokin@mcw.edu.

Jozef Lazar and Howard J. Jacob's present address is: HudsonAlpha Institute for Biotechnology, Huntsville, Alabama, USA.

Richard J. Roman and Jan M. Williams's present address is: Department of Pharmacology and Toxicology, University of Mississippi Medical Center, Jackson, Mississippi, USA. 
1. Briones AM, Touyz RM. Oxidative stress and hypertension: current concepts. Curr Hypertens Rep. 2010;12(2):135-142.

2. Rodriguez-Iturbe B, Johnson RJ. The role of renal microvascular disease and interstitial inflammation in salt-sensitive hypertension. Hypertens Res. 2010;33(10):975-980.

3. Migliaccio E, et al. The p66shc adaptor protein controls oxidative stress response and life span in mammals. Nature. 1999;402(6759):309-313.

4. Francia P, et al. Deletion of p66shc gene protects against age-related endothelial dysfunction. Circulation. 2004;110(18):2889-2895.

5. Purdom S, Chen QM. p66(Shc): at the crossroad of oxidative stress and the genetics of aging. Trends Mol Med. 2003;9(5):206-210.

6. Barton M, Lattmann T, d'Uscio LV, Lüscher TF, Shaw S. Inverse regulation of endothelin- 1 and nitric oxide metabolites in tissue with aging: implications for the age-dependent increase of cardiorenal disease. J Cardiovasc Pharmacol. 2000;36(5 suppl 1):S153-S156.

7. Foschi M, Franchi F, Han J, La Villa G, Sorokin A. Endothelin-1 induces serine phosphorylation of the adaptor protein p66Shc and its association with 14-3-3 protein in glomerular mesangial cells. J Biol Chem. 2001;276(28):26640-26647.

8. Campese VM. Salt sensitivity in hypertension. Hypertension. 1994;23(4):531-550.

9. Cowley AW Jr, et al. Brown Norway chromosome 13 confers protection from high salt to consomic Dahl S rat. Hypertension. 2001; 37(2 pt 2):456-461.

10. Mori T, et al. High perfusion pressure accelerates renal injury in salt-sensitive hypertension. $J \mathrm{Am}$ Soc Nephrol. 2008;19(8):1472-1482.

11. Taylor NE, Glocka P, Liang M, Cowley AW. NADPH oxidase in the renal medulla causes oxidative stress and contributes to salt-sensitive hypertension in Dahl S rats. Hypertension. 2006;47(4):692-698.

12. Cowley AW Jr. The genetic dissection of essential hypertension. Nat Rev Genet. 2006;7(11):829-840.

13. Cowley AW, Liang M, Roman RJ, Greene AS, Jacob HJ. Consomic rat model systems for physiological genomics. Acta Physiol Scand. 2004;181(4):585-592.

14. Imig JD, Inscho EW. Adaptations of the renal microcirculation to hypertension. Microcirculation. 2002;9(4):315-328.

15. Zhao X, et al. Impaired $\mathrm{Ca}^{2+}$ signaling attenuates $\mathrm{P} 2 \mathrm{X}$ receptor-mediated vasoconstriction of afferent arterioles in angiotensin II hypertension. Hypertension. 2005;46(3):562-568.

16. Inscho EW, Cook AK, Imig JD, Vial C, Evans RJ. Physiological role for $\mathrm{P} 2 \mathrm{X} 1$ receptors in renal microvascular autoregulatory behavior. J Clin Invest. 2003;112(12):1895-1905.

17. Inscho EW, Cook AK, Imig JD, Vial C, Evans RJ. Renal autoregulation in P2X1 knockout mice. Acta Physiol Scand. 2004;181(4):445-453.

18. Inscho EW, Ohishi K, Navar LG. Effects of ATP on pre- and postglomerular juxtamedullary microvasculature. Am J Physiol. 1992; 263(5 pt 2):F886-F893.
19. Ventura A, Luzi L, Pacini S, Baldari CT, Pelicci PG. The p66Shc longevity gene is silenced through epigenetic modifications of an alternative promoter. J Biol Chem. 2002;277(25):22370-22376.

20. Johnson AA, Akman K, Calimport SR, Wuttke D, Stolzing A, de Magalhães JP. The role of DNA methylation in aging, rejuvenation, and age-related disease. Rejuvenation Res. 2012;15(5):483-494.

21. Lai KM, Pawson T. The ShcA phosphotyrosine docking protein sensitizes cardiovascular signaling in the mouse embryo. Genes Dev. 2000;14(9):1132-1145.

22. Geurts AM, et al. Knockout rats via embryo microinjection of zinc-finger nucleases. Science. 2009;325(5939):433.

23. Burke M, Pabbidi MR, Farley J, Roman RJ. Molecular mechanisms of renal blood flow autoregulation. Curr Vasc Pharmacol. 2014;12(6):845-858.

24. Guan Z, Inscho EW. Endothelin and the renal vasculature. Contrib Nephrol. 2011;172:35-49.

25. Adamczak M, et al. Reversal of glomerulosclerosis after high-dose enalapril treatment in subtotally nephrectomized rats. J Am Soc Nephrol. 2003;14(11):2833-2842.

26. Kohan DE, Rossi NF, Inscho EW, Pollock DM. Regulation of blood pressure and salt homeostasis by endothelin. Physiol Rev. 2011; 91(1):1-77.

27. Rautureau Y, Schiffrin EL. Endothelin in hypertension: an update. Curr Opin Nephrol Hypertens. 2012;21(2):128-136

28. Sorokin A, Kozlowski P, Graves L, Philip A. Protein-tyrosine kinase Pyk2 mediates endothelin-induced p38 MAPK activation in glomerular mesangial cells. J Biol Chem. 2001;276(24):21521-21528.

29. Gerthoffer WT, Gunst SJ. Invited review: focal adhesion and small heat shock proteins in the regulation of actin remodeling and contractility in smooth muscle. J Appl Physiol. 2001;91(2):963-972.

30. Guyton AC. Blood pressure control - special role of the kidneys and body fluids. Science. 1991;252(5014):1813-1816.

31. Imig JD. Afferent arteriolar reactivity to angiotensin II is enhanced during the early phase of angiotensin II hypertension. Am J Hypertens. 2000;13(7):810-818.

32. Kost CK, Jackson EK. Enhanced renal angiotensin II subtype 1 receptor responses in the spontaneously hypertensive rat. Hypertension. 1993;21(4):420-431.

33. Schiffrin EL. Intracellular signal transduction for vasoactive peptides in hypertension. Can J Physiol Pharmacol. 1994;72(8):954-962.

34. Smith L, Payne JA, Sedeek MH, Granger JP, Khalil RA. Endothelin-induced increases in $\mathrm{Ca}^{2+}$ entry mechanisms of vascular contraction are enhanced during high-salt diet. Hypertension. 2003;41(3 pt 2):787-793.

35. Inscho EW, Cook AK, Murzynowski JB, Imig JD. Elevated arterial pressure impairs autoregulation independently of AT(1) receptor activation. J Hypertens. 2004;22(4):811-818.
36. Amiri F, Garcia R. Renal angiotensin II receptor regulation in two-kidney, one clip hypertensive rats: effect of ACE inhibition. Hypertension. 1997;30(3 pt 1):337-344.

37. Chatziantoniou C, Arendshorst WJ. Angiotensin receptor sites in renal vasculature of rats developing genetic hypertension. Am J Physiol. 1993;265(6 pt 2):F853-F862.

38. Sgouralis I, Layton AT. Autoregulation and conduction of vasomotor responses in a mathematical model of the rat afferent arteriole. Am J Physiol Renal Physiol. 2012;303(2):F229-F239.

39. Hayashi K, Epstein M, Saruta T. Altered myogenic responsiveness of the renal microvasculature in experimental hypertension. J Hypertens. 1996;14(12):1387-1401.

40. Sorokin A. Endothelin signaling and actions in the renal mesangium. Contrib Nephrol. 2011;172:50-62.

41. Chahdi A, Sorokin A. Endothelin-1 couples $\beta$ Pix to p66Shc: role of betaPix in cell proliferation through FOXO3a phosphorylation and p27kip1 down-regulation independently of Akt. Mol Biol Cell. 2008;19(6):2609-2619.

42. Chahdi A, Sorokin A. Endothelin-1 induces p66Shc activation through EGF receptor transactivation: role of $\beta(1) \mathrm{Pix} / \mathrm{G} \alpha(\mathrm{i} 3)$ interaction. Cell Signal. 2010;22(2):325-329.

43. Palygin O, Miller B, Ilatovskaya DV, Sorokin A, Staruschenko A. Two-photon imaging of endothelin-1-mediated intracellular $\mathrm{Ca}^{2+}$ handling in smooth muscle cells of rat renal resistance arteries [published online ahead of print December 10, 2015]. Life Sci. doi:10.1016/j.lfs.2015.12.022.

44. Facemire CS, Mohler PJ, Arendshorst WJ. Expression and relative abundance of short transient receptor potential channels in the rat renal microcirculation. Am J Physiol Renal Physiol. 2004;286(3):F546-F551.

45. Gonzalez-Cobos JC, Trebak M. TRPC channels in smooth muscle cells. Front Biosci. 2010;15:1023-1039.

46. Saleh SN, Albert AP, Large WA. Activation of native TRPC1/C5/C6 channels by endothelin-1 is mediated by both PIP3 and PIP2 in rabbit coronary artery myocytes. J Physiol (Lond). 2009;587(pt 22):5361-5375.

47. Salomonsson M, Braunstein TH, HolsteinRathlou NH, Jensen LJ. $\mathrm{Na}^{+}$-independent, nifedipine-resistant rat afferent arteriolar $\mathrm{Ca} 2+$ responses to noradrenaline: possible role of TRPC channels. Acta Physiol (Oxf). 2010;200(3):265-278

48. Fellner SK, Arendshorst WJ. Angiotensin II-stimulated Ca2+ entry mechanisms in afferent arterioles: role of transient receptor potential canonical channels and reverse $\mathrm{Na}^{+} /$ $\mathrm{Ca}^{2+}$ exchange. Am J Physiol Renal Physiol. 2008;294(1):F212-F219.

49. Nishiyama A, Rahman M, Inscho EW. Role of interstitial ATP and adenosine in the regulation of renal hemodynamics and microvascular function. Hypertens Res. 2004;27(11):791-804.

50. Abboud HE. Mesangial cell biology. Exp Cell Res. 2012;318(9):979-985

51. Kotchen TA, Cowley AW Jr, Frohlich ED. Salt in health and disease - a delicate balance. $N$ Engl J 
Med. 2013;368(13):1229-1237.

52. Lackland DT, Keil JE. Epidemiology of hypertension in African Americans. Semin Nephrol. 1996;16(2):63-70.

53. Schiffrin EL. Role of endothelin-1 in hypertension and vascular disease. Am J Hypertens. 2001;14(6 pt 2):83S-89S.

54. Ito $\mathrm{S}$. Cardiorenal syndrome: an evolutionary point of view. Hypertension. 2012; 60(3):589-595.

55. Imig JD, Dimitropoulou C, Reddy DS, White RE, Falck JR. Afferent arteriolar dilation to 11, 12-EET analogs involves PP2A activity and $\mathrm{Ca}^{2+}$-activated $\mathrm{K}^{+}$channels. Microcirculation. 2008;15(2):137-150.

56. Geurts AM, et al. Generation of gene-specific mutated rats using zinc-finger nucleases. Methods Mol Biol. 2010;597:211-225.

57. Mattson DL, Lund H, Guo C, Rudemiller N, Geurts AM, Jacob H. Genetic mutation of recombination activating gene 1 in Dahl saltsensitive rats attenuates hypertension and renal damage. Am J Physiol Regul Integr Comp Physiol. 2013;304(6):R407-R414. 